\title{
Abiraterone acetate post-docetaxel for metastatic castration-resistant prostate cancer in the Belgian compassionate use program
}

\author{
Charles Van Praet, M.D. ${ }^{\mathrm{a}, *}$, Sylvie Rottey, M.D., Ph.D. ${ }^{\mathrm{b}}$, Fransien Van Hende, M.D. ${ }^{\mathrm{c}}$,
} Gino Pelgrims, M.D. ${ }^{\mathrm{d}}$, Wim Demey, M.D. ${ }^{\mathrm{e}}$, Filip Van Aelst, M.D. ${ }^{\mathrm{f}}$, Wim Wynendaele, M.D. ${ }^{\mathrm{g}}$, Thierry Gil, M.D. , Peter Schatteman, M.D. ${ }^{\mathrm{i}}$, Bertrand Filleul, M.D. ${ }^{\mathrm{j}}$, Dennis Schallier, M.D., Ph.D. , Jean-Pascal Machiels, M.D., Ph.D. ${ }^{1}$, Dirk Schrijvers, M.D. ${ }^{\mathrm{m}}$, Els Everaert, M.D. ,

Lionel D'Hondt, M.D. ${ }^{\circ}$, Patrick Werbrouck, M.D. ${ }^{p}$, Joanna Vermeij, M.D. ${ }^{q}$,

Jeroen Mebis, M.D. ${ }^{r}$, Marylene Clausse, M.D. , Marika Rasschaert, M.D. ${ }^{\mathrm{s}}$, Joanna Van Erps, M.D. ${ }^{u}$, Jolanda Verheezen, M.D. ", Jan Van Haverbeke, M.D. ${ }^{\mathrm{w}}$, Jean-Charles Goeminne, M.D. ${ }^{\mathrm{x}}$, Nicolaas Lumen, M.D., Ph.D. ${ }^{\mathrm{a}}$

\author{
${ }^{a}$ Department of Urology, Ghent University Hospital, Ghent, Belgium \\ ${ }^{\mathrm{b}}$ Department of Medical Oncology, Ghent University Hospital, Ghent, Belgium \\ ${ }^{\mathrm{c}}$ Department of Medical Oncology, University Hospital Leuven, Leuven, Belgium \\ ${ }^{\mathrm{d}}$ Department of Medical Oncology, AZ Turnhout, Turnhout, Belgium \\ ${ }^{\mathrm{e}}$ Department of Medical Oncology, AZ Klina, Kalmthout, Belgium \\ ${ }^{\mathrm{f}}$ Department of Medical Oncology, AZ Delta, Roeselare, Belgium \\ ${ }^{\mathrm{g}}$ Department of Medical Oncology, Imelda Hospital, Bonheiden, Belgium \\ ${ }^{\mathrm{h}}$ Department of Medical Oncology, Institut Jules Bordet, Université Libre de Bruxelles, Brussels, Belgium \\ ${ }^{i}$ Department of Urology, Onze-Lieve-Vrouw Hospital, Aalst, Belgium \\ ${ }^{\mathrm{j}}$ Department of Medical Oncology, Hopital De Jolimont, Haine Saint Paul, Belgium \\ ${ }^{\mathrm{k}}$ Department of Medical Oncology, University Hospital Brussels, Jette, Belgium \\ ${ }^{1}$ Department of Medical Oncology, Institut Roi Albert II, Cliniques Universitaires Saint Luc, Brussels, Belgium \\ ${ }^{\mathrm{m}}$ Department of Medical Oncology, Ziekenhuis Netwerk Antwerpen (ZNA) Middelheim, Antwerp, Belgium \\ ${ }^{\mathrm{n}}$ Department of Medical Oncology, AZ Nikolaas, Sint-Niklaas, Belgium \\ ${ }^{\circ}$ Department of Medical Oncology, CHU Dinant-Godinne, Yvoir, Belgium \\ ${ }^{\mathrm{p}}$ Department of Urology, AZ Groeninge, Kortrijk, Belgium \\ ${ }^{\mathrm{q}}$ Department of Medical Oncology, ZNA Jan Palfijn, Merksem, Belgium \\ ${ }^{\mathrm{r}}$ Department of Medical Oncology, AZ Jessa, Hasselt, Belgium \\ ${ }^{\mathrm{s}}$ Department of Medical Oncology, St Luc Bouge, Namur, Belgium \\ ${ }^{\mathrm{t}}$ Department of Medical Oncology, UZA/AZ Monica, Antwerp, Belgium \\ ${ }^{\mathrm{u}}$ Department of Medical Oncology, Algemeen Stedelijk Ziekenhuis, Aalst, Belgium \\ ${ }^{v}$ Department of Medical Oncology, AZ Sint-Trudo, Sint-Truiden, Belgium \\ ${ }^{\mathrm{w}}$ Department of Urology, AZ Sint-Andries, Tielt, Belgium \\ ${ }^{\mathrm{x}}$ Department of Medical Oncology, Sainte-Elisabeth, Namur, Belgium
}

Received 25 October 2015; accepted 28 December 2015

\footnotetext{
Janssen provided financial support for the conduct of this research. Janssen did not have an active part in study design, in the collection, analysis and interpretation of the data, in the writing of the report, or in the decision to submit the article for publication. Charles Van Praet is consultant for Janssen; Sylvie Rottey received grants and honoraria from Sanofi Jean-Pascal Machiels received grants from Janssen and Novartis; Dirk Schrijvers is consultant for Janssen; Els Everaert is consultant for Janssen; Patrick Werbrouck is consultant for Bayer and Janssen; Nicolaas Lumen is consultant for Bayer, Ipsen, Janssen, and Lilly and received grants from Astellas, Astra Zeneca, Janssen, and Sanofi.

${ }^{*}$ Corresponding author. Tel.: +32-47-376-8176; fax: +32-93-32-38-89.

E-mail addresses: charles.vanpraet@uzgent.be, vanpraetcharles@gmail.com (C. Van Praet).
} 


\begin{abstract}
Background: Abiraterone acetate (AA) is licensed for treating metastatic castration-resistant prostate cancer (mCRPC). Real-world data on oncological outcome after AA are scarce. The current study assesses efficacy and safety of AA in mCRPC patients previously treated with docetaxel who started treatment during the Belgian compassionate use program (January 2011-July 2012).

Patients and methods: Records from 368 patients with mCRPC from 23 different Belgian hospitals who started AA 1000 mg per day with $10 \mathrm{mg}$ prednisone or equivalent were retrospectively reviewed (September 2013-December 2014). Prostate-specific antigen (PSA) response (decrease $\geq 50 \%$ ), time to PSA progression (increase $>50 \%$ over PSA nadir in case of PSA response/ $>25 \%$ in absence of PSA response), time to radiographic progression (on bone scans or for soft tissue lesions using Response Evaluation Criteria In Solid Tumors 1.1), overall survival and adverse event rate (Common Terminology Criteria for Adverse Events v4.03) were analyzed. Kaplan-Meier statistics were applied.

Results: Overall, 92 patients $(25 \%)$ had an Eastern Cooperative Oncology Group performance status $\geq 2$. Median age was 73 years, median PSA was $103 \mathrm{ng} / \mathrm{dl}$. PSA response was observed in 131 patients (37.4\%). Median time to PSA and radiographic progression was 4.1 months (95\% CI: 3.6-4.6) and 5.8 months (5.3-6.4), respectively. Median overall survival was 15.1 months (13.6-16.6). Most common grade 3 to 4 adverse events were anemia (13.9\%), hypokalemia (7.3\%), fatigue (6.8\%), and pain (6.3\%). Median duration of AA treatment was 5.3 months (interquartile range: $2.8-10.3$ ). The main study limitation is its retrospective design.

Conclusions: These real-world data on post-docetaxel AA efficacy are in line with the COU-AA-301 trial. Importantly, incidence of severe anemia and hypokalemia is up to 50\% higher than reported in previous studies. (C) 2016 Elsevier Inc. All rights reserved.
\end{abstract}

Keywords: Prostatic neoplasms; Castration-resistant; Abiraterone acetate; Compassionate use; Docetaxel

\section{Introduction}

Since 2010, several new drugs have become available for treatment of metastatic castration-resistant prostate cancer (mCRPC) following progression on docetaxel [1-4]. Abiraterone acetate (AA) was one of the first to demonstrate clinical efficacy in its phase 3 trial, COU-AA-301 [1]. Later, COU-AA-302 also demonstrated clinical efficacy in patients with chemotherapy-naive mCRPC [5]. AA is a prodrug of abiraterone, a potent inhibitor of CYP17 enzymes, thus inhibiting adrenal and intratumoral androgen synthesis. In COU-AA-301, patients on AA $1000 \mathrm{mg}$ + prednisone $10 \mathrm{mg}$ daily showed superior overall survival (OS) compared with patients receiving placebo + prednisone $10 \mathrm{mg}$ daily (15.8 mo vs. $11.2 \mathrm{mo}$, respectively), therefore achieving its goal of demonstrating superior clinical efficacy [1]. However, because of its focus on efficacy and standardized protocols for patient selection and follow-up, its results might not necessarily translate into efficacy in daily clinical practice [6,7]. Little is known about the real-world efficacy and adverse event rate of AA. To our knowledge only 3 studies have analyzed oncological outcome and tolerability in a cohort of $>100$ patients with mCRPC post-docetaxel outside clinical trials [8-10]. Therefore, we retrospectively analyzed patients with mCRPC who started AA treatment in the Belgian compassionate use (CU) program (January 2011-July 2012). The goal of this article is to determine oncological outcome and adverse event rate and indirectly compare these with the active arm of COU-AA-301. To the best of our knowledge, this article reports results from the largest mCRPC cohort outside a clinical trial.

\section{Materials and methods}

\subsection{Patients}

Physicians from 23 Belgian hospitals provided a list of all consecutive patients who started AA treatment in the Belgian CU program. A total of 368 patients were identified. This CU program was initiated on January 1, 2011, allowing patients with docetaxel-treated mCRPC access to AA. It closed July 31, 2012, when AA was reimbursed for use post-docetaxel in Belgium. All patients started AA at a dose of $1000 \mathrm{mg}$ daily with $10 \mathrm{mg}$ prednisone or equivalent. Treatment was continued until disease progression, severe toxicity, death or patient/physician's preference. At the end of the Belgian CU program, patients still on AA continued this treatment. After AA start, patient follow-up was at the treating physician's discretion, but follow-up visits were recommended every 2 weeks for the first 3 months and then monthly thereafter for safety, with prostate-specific antigen (PSA) levels and radiographic evaluation assessed every 3 months.

Data on oncological outcome, adverse events, and previous prostate cancer (PC) treatments were retrospectively retrieved from the patient file by the first author (C.V.P.) in 18 centers (264 patients; 71.7\%) or by local data managers in 5 centers (104 patients; 28.3\%) between September 2013 and December 2014. Data retrieved included all available reports from clinical visits, lab results, imaging, and date of death. Follow-up time was defined as time between start of AA and date of last follow-up or death. PSA doubling time was calculated using the Memorial Sloan Kettering online calculator [11]. Date of 
CRPC was defined as date of PSA progression following surgical or chemical castration therapy or anti-androgen therapy before docetaxel. The study was approved by central and local ethic committees (central EC UZG 2013/413).

Patients were eligible for the CU program when they had histologically/cytologically confirmed PC, received at least 1 but no more than 2 different cytotoxic chemotherapy regimens for mCRPC with at least 1 taxane regimen and had PC progression, defined by PSA progression according to Prostate Cancer Working Group 2 criteria [12] or by radiographic progression in soft tissue according to Response Evaluation Criteria in Solid Tumors criteria version 1.1, [13] or on bone scans. Patients were to have a serum testosterone $<50 \mathrm{ng} / \mathrm{dl}$, an Eastern Cooperative Oncology Group (ECOG) performance status $\leq 2$, hemoglobin $\geq 9.0 \mathrm{~g} / \mathrm{dl}$, platelet count $\geq 100.000 / \mu \mathrm{l}$, serum albumin $\geq 3.0 \mathrm{~g} / \mathrm{dl}$, serum creatinine $<1.5 \times$ upper limit of normal (ULN) or a calculated creatinine clearance $\geq 60$ $\mathrm{ml} / \mathrm{min}$ and serum potassium $\geq 3.5 \mathrm{mmol} / \mathrm{l}$.

Patients were ineligible for the $\mathrm{CU}$ program if they were previously enrolled in COU-AA-301 or COU-AA-302, had serious or uncontrolled co-existent nonmalignant disease, had bilirubin $\geq 1.5 \times$ ULN or aspartate aminotransferase, or alanine aminotransferase $\geq 2.5 \times \mathrm{ULN}$, uncontrolled hypertension, active or symptomatic viral hepatitis or chronic liver disease, history of pituitary or adrenal dysfunction, clinically significant heart disease, known brain metastasis or any acute toxicities because of prior chemotherapy or radiotherapy that had not resolved to a grade $\leq 1$ according to Common Terminology Criteria for Adverse Events.

\subsection{Outcomes}

Primary outcome was OS, defined as time from start of AA until death. Secondary outcomes were cancer-specific survival (CSS), PSA response rate, time to PSA progression, time to radiographic progression and rate of adverse events, skeletal-related events (SREs) and number of hospitalizations during AA treatment. CSS was defined as time from start of AA until death because of PC. A PSA response was defined as a PSA decline $\geq 50 \%$ after at least 4 weeks of AA treatment, confirmed by a second PSA decline at least 4 weeks later. Similar to COU-AA-301, for patients whose PSA did not decrease, PSA progression was defined as a PSA increase $\geq 25 \%$ over the baseline and an increase $\geq 2 \mathrm{ng} / \mathrm{ml}$. For patients whose PSA decreased $<50 \%$, PSA progression was defined as a PSA increase $\geq 25 \%$ above the nadir and an increase $\geq 2$ $\mathrm{ng} / \mathrm{ml}$. For PSA responders, PSA progression was defined as a PSA increase $\geq 50 \%$ above the nadir and an increase $\geq 2 \mathrm{ng} / \mathrm{ml}$. To allow more accurate comparison with COU-AA-301 data, secondary calculations were made for PSA response and time to PSA progression using only PSA values measured at 3-monthly intervals. Radiographic progression was defined as progression by soft tissue imaging (modified response evaluation criteria in solid tumors criteria with a baseline lymph node $\geq 2.0 \mathrm{~cm}$ to be considered target lesion [13]) or by bone scan with $\geq 2$ new lesions not consistent with tumor flare. Analysis of radiographic progression was restricted to patients who had imaging (computed tomography, magnetic resonance imaging, or bone scan) within 30 days before AA start. The following adverse events during AA treatment were scored according to Common Terminology Criteria for Adverse Events version 4.03 [14]: pain, diarrhea, nausea, vomiting, fatigue, peripheral edema, cardiac disorders (ischemic heart disease; myocardial infarction; [supra] ventricular tachyarrhythmias; cardiac failure; or possible arrhythmia-related investigations, signs, and symptoms), anemia, neutropenia, thrombocytopenia, rising liver enzymes (aspartate aminotransferase or alanine aminotransferase), hypokalemia and hypertension (at least 3 blood pressure measurements required). SRE was defined as any pathological bone fracture, spinal cord compression, palliative radiation to the bone or surgery to the bone because of bone metastases.

\subsection{Statistical analysis}

Continuous variables are presented as median (interquartile range [IQR]). Survival analyses were performed using Kaplan-Meier statistics. Differences between categorical variables were assessed using chi-square test. All statistical analyses were performed with SPSS version 22.0 (IBM, NY). $P<0.05$ was considered statistically significant.

\section{Results}

\subsection{Patient characteristics and AA treatment}

Patient, disease and previous treatment characteristics are listed in Table 1. CU protocol violations included 5 (1.4\%) patients who received no previous chemotherapy, 23 (6.3\%) patients who previously received $>2$ different chemotherapy regimens and $9(2.4 \%)$ patients who had an ECOG performance status $\geq 3$ at start of AA. These patients were included in further analysis. Median duration of AA treatment was 5.3 months (IQR: 2.8-10.3). After a median follow-up time of 13.9 months (IQR: 6.1-22.6), 334 (90.8\%) patients discontinued AA, either because of disease progression $(276,75.0 \%)$, toxicity $(29,7.9 \%)$, death $(28 ; 7.6 \%)$, or physician's preference $(1,0.3 \%)$. AA treatment was still ongoing in 17 (4.6\%) patients, whereas data on AA continuation were missing in 17 (4.6\%) patients at the time of evaluation. Treatment with AA was temporarily interrupted in $15(4.1 \%)$ patients, whereas a dose reduction to 500 to $750 \mathrm{mg}$ AA daily was performed in 13 (3.5\%) patients, all related to toxicity. 
Table 1

Patient, disease and previous treatment characteristics

\begin{tabular}{|c|c|c|}
\hline & $N=368$ & $\begin{array}{l}\text { Missing } \\
\text { cases }\end{array}$ \\
\hline \multicolumn{3}{|l|}{ Demographics at start AA } \\
\hline Age, years, median (IQR) & $73(66-77)$ & 2 \\
\hline ECOG performance status, $n(\%)$ & & $13(3.5)$ \\
\hline $0-1$ & $263(71.5)$ & \\
\hline 2 & $83(22.6)$ & \\
\hline 3 & $7(1.9)$ & \\
\hline 4 & $2(0.5)$ & \\
\hline \multicolumn{3}{|l|}{ Serum values at start AA, median (IQR) } \\
\hline Hemoglobin, g/dl & $11.6(10.2-13.0)$ & 19 \\
\hline Alkaline phosphatase, U/l & $142(80-287)$ & 36 \\
\hline Lactate dehydrogenase, $\mathrm{U} / \mathrm{l}$ & $513(379-692)$ & 48 \\
\hline Prostate-specific antigen, $\mathrm{ng} / \mathrm{dl}$ & $103(34-329)$ & 4 \\
\hline \multicolumn{3}{|l|}{ Time parameters at start AA, median (IQR) } \\
\hline Time since diagnosis, mo & $77(41-129)$ & 45 \\
\hline Time since CRPC, mo & $22(11-40)$ & 129 \\
\hline Time off treatment, wk & $11(4-22)$ & 31 \\
\hline \multicolumn{3}{|l|}{ Disease characteristics at start AA, $n(\%)$} \\
\hline Local tumor in prostate (bed) & $183(49.7)$ & $27(7.3)$ \\
\hline Lymph node metastases & $205(55.7)$ & $15(4.1)$ \\
\hline Bone metastases & $327(88.9)$ & $5(1.4)$ \\
\hline Visceral metastases (all) & 76 (20.7) & $12(3.3)$ \\
\hline Liver metastases & $32(8.7)$ & $12(3.3)$ \\
\hline Lung metastases & $31(8.4)$ & $12(3.3)$ \\
\hline Disease progression & & $74(20.1)$ \\
\hline PSA only & $94(25.5)$ & \\
\hline progression on imaging & $200(54.3)$ & \\
\hline Presence of pain & $250(67.9)$ & $16(4.3)$ \\
\hline \multicolumn{3}{|l|}{ Previous treatment characteristics, $n(\%)$} \\
\hline $\begin{array}{l}\text { Local treatment with surgery or } \\
\text { radiotherapy }\end{array}$ & $210(57.1)$ & $18(4.9)$ \\
\hline Number of chemotherapy lines ${ }^{\mathrm{a}}$ & & $12(3.3)$ \\
\hline 0 & $5(1.4)$ & \\
\hline 1 & $180(48.9)$ & \\
\hline 2 & $109(29.6)$ & \\
\hline 3 & $44(12.0)$ & \\
\hline$>3$ & $18(4.9)$ & \\
\hline Bone protecting agent before start AA & $245(66.6)$ & $20(5.4)$ \\
\hline Prior skeletal-related event & $135(36.7)$ & $42(11.4)$ \\
\hline
\end{tabular}

${ }^{\mathrm{a}}$ Rechallenge with a chemotherapy line previously administered was considered as another line.

\subsection{Oncological outcome}

Of all 368 patients, $283(76.9 \%)$ died-213 (57.9\%) because of PC progression, 10 (2.7\%) because of infectious disease, $3(0.8 \%)$ because of another malignancy, and 7 $(1.9 \%)$ because of other causes. In $50(13.6 \%)$ patients, cause of death was unknown. Median OS and CSS from start of AA were 15.1 months (95\% CI: 13.6-16.6) and 16.8 months (95\% CI: 14.6-19.0), respectively. Median OS from date of CRPC was 42.0 months (95\% CI: 34.6-49.5) (based on 237 patients with 179 events). In 18 (4.9\%) patients, no PSA values during AA treatment were available. Of the remaining 350 patients, 131 (37.4\%) had a PSA response (Fig. 1). PSA progression was documented in $280(80.0 \%)$ patients. Median time to PSA progression was 4.1 months (95\% CI: 3.6-4.6). When only using 3 monthly PSA values, as was customary in COU-AA-301, usable PSA values were missing in $57(15.5 \%)$ patients. Of the remaining 311 patients, $103(33.1 \%)$ had a PSA response and median time to PSA progression was 6.0 months (95\% CI: 5.5-6.5). In an intention-to-treat analysis in patients with any PSA follow-up, 29.4\% (103 of 350) had a PSA response and median time to PSA progression was 6.0 months (95\% CI: 5.5-6.5). Only $212(57.6 \%)$ patients had imaging before AA start, of which 119 (56.1\%) experienced radiographic progression. Median time to radiographic progression was 5.8 months (95\% CI: 5.3-6.4) (Fig. 2).

\subsection{Adverse events}

Rate of grade 3 to 4 hematological disorders (anemia, neutropenia, and thrombocytopenia) and hypokalemia was notably high (Table 2). Patients with an ECOG performance status $\geq 2$ were more susceptible to hematological disorders and hypokalemia (all $P<0.05$ ). Overall, 68 SREs occurred during $\mathrm{AA}$ in $57(15.5 \%)$ patients as follows: 50 cases of palliative radiotherapy, 12 observations of spinal cord compression, 4 cases of bone fracture, and 2 patients who had surgery to the bone. Finally, 137 (37.3\%) patients were hospitalized at least once during AA treatment.

\section{Discussion}

We retrospectively assessed oncological outcome and adverse events of AA treatment post-docetaxel in 368 patients with mCRPC from 23 hospitals during the Belgian $\mathrm{CU}$ program. Although our study is limited by its retrospective design, our oncological results confirm AA's efficacy [1]. Regarding tolerability, however, concerns can be raised over a surprisingly high incidence of hematological side effects and hypokalemia. To the best of our knowledge, this is the largest cohort of patients with taxane pretreated mCRPC reported on in a nonclinical trial setting (Table 3). Previously, Caffo et al. [8] retrospectively analyzed records from 265 patients with mCRPC treated during the Italian named patient program. Clayton et al. [9] retrospectively analyzed 187 patients with mCRPC treated at 5 tertiary Canadian centers. More recently, Houédé et al. [10] retrospectively analyzed 306 patients with mCRPC treated during the French temporary authorization for use program. These studies reported good clinical outcome and low adverse event rate. In a worldwide early access protocol study in 2314 patients, Sternberg et al. [15] reported low adverse event rate. Furthermore, Azad et al. [16] described a cohort of 519 patients, both chemotherapy naïve and pretreated, who received AA, but only reported on different oncological outcome between patients with ECOG $<2$ and $\mathrm{ECOG} \geq 2$. 


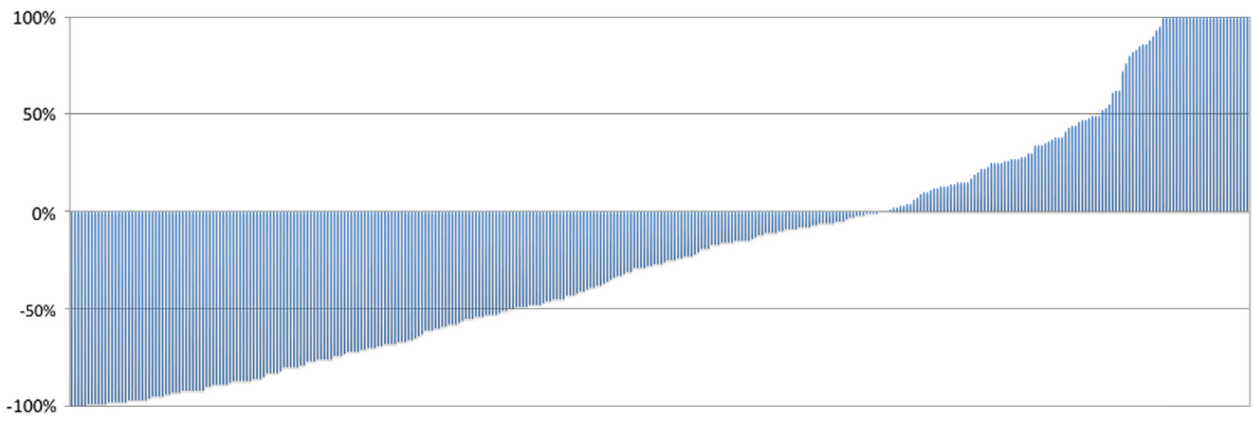

Fig. 1. Maximal PSA drop during abiraterone acetate treatment. Waterfall plot depicting maximal prostate-specific antigen (PSA) response (\%) for each patient, based on the formula (PSA nadir during abiraterone acetate [AA]—PSA at start AA)/PSA at start AA. Negative values indicate a drop in PSA. All available PSA measurements for each patient were used to determine PSA nadir. (Color version of figure is available online.)

In our series, rate of anemia $(91.6 \%$ all grades, $13.9 \%$ grade $\geq 3)$ and hypokalemia $(7.3 \%$ grade $\geq 3)$ was higher compared with COU-AA-301 $(7.8 \%$ and $4.4 \%$ grade $\geq 3$,

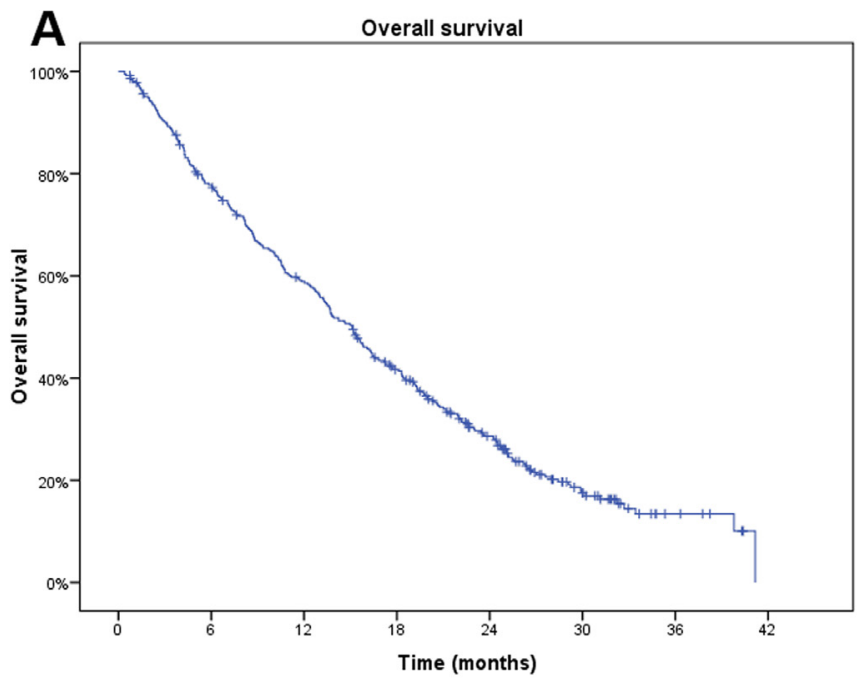

$\begin{array}{llllllll}\text { Number at risk } 366 & 278 & 207 & 139 & 80 & 32 & 7 & 0\end{array}$

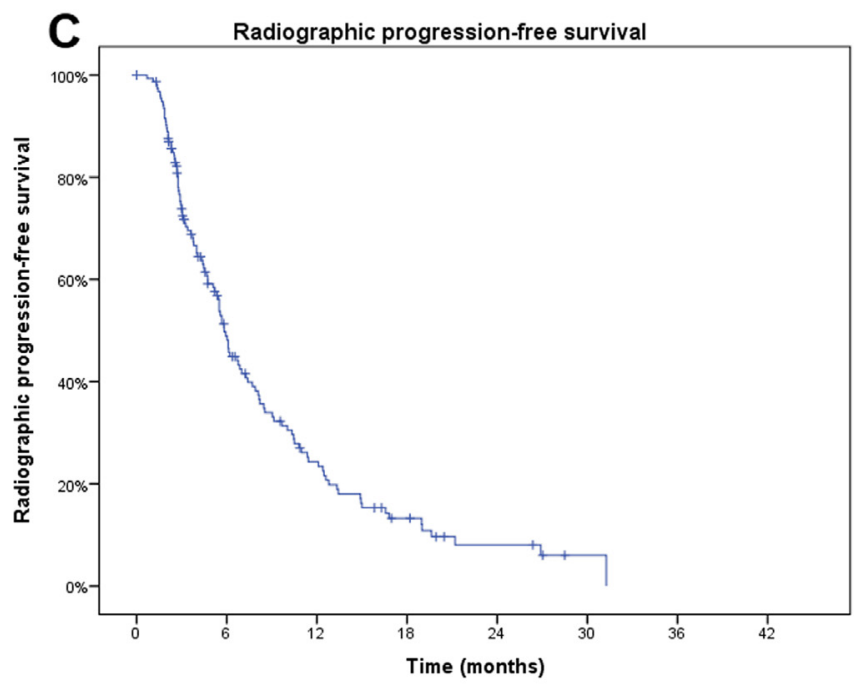

Number at risk $\quad 210 \quad 61 \quad 27$ respectively) and other reports $(0.1 \%-4.2 \%$ and $0.4 \%-1.6 \%$ respectively, Table 2) $[1,8-10,15]$. We have several possible explanations. First, patients in clinical trials are generally in
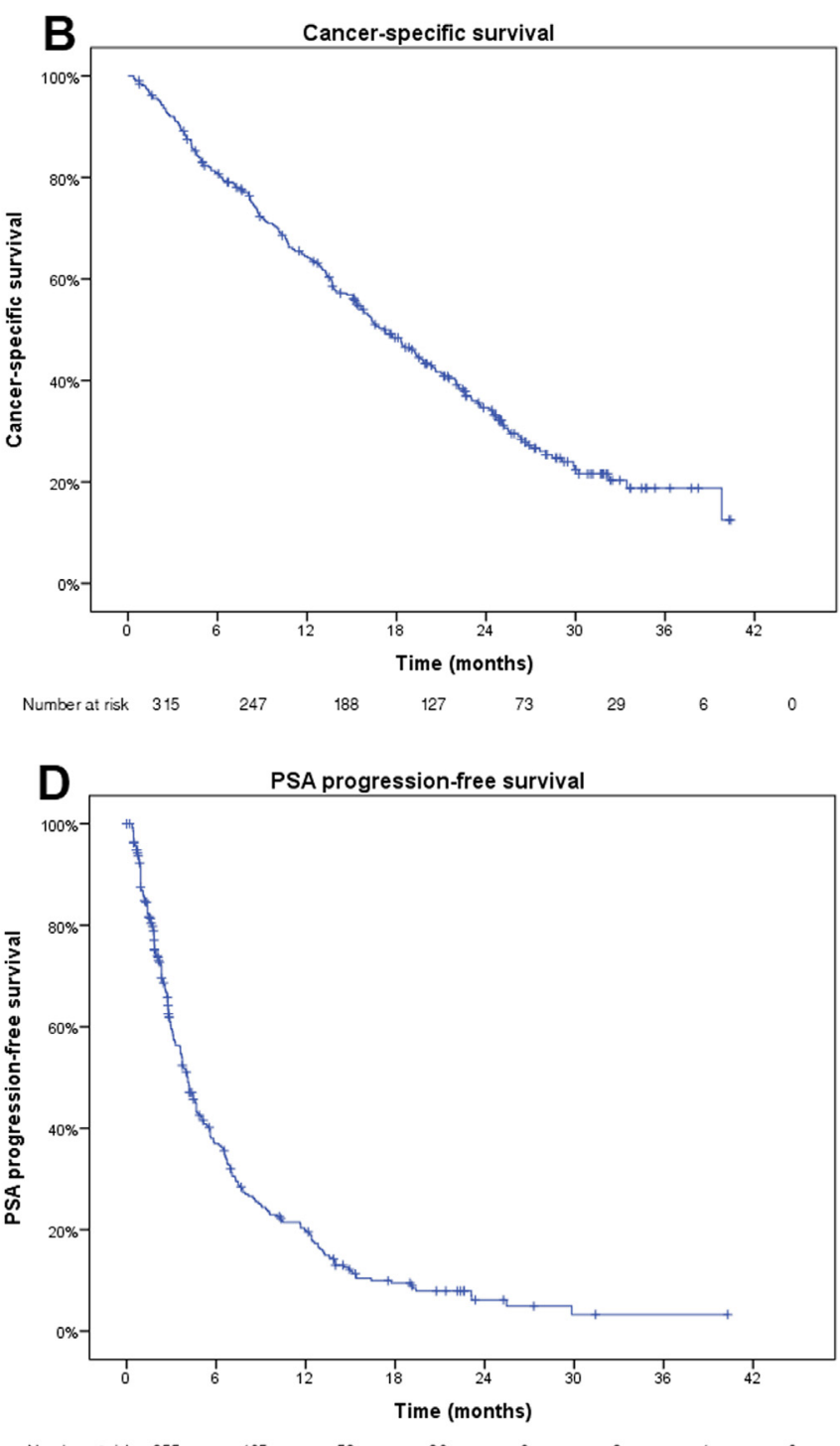

$\begin{array}{llllllll}\text { Number at risk } & 355 & 105 & 52 & 20 & 6 & 2 & 1\end{array}$

Fig. 2. Patient outcomes. Kaplan-Meier plots depicting overall survival (A), cancer-specific survival (B), radiographic progression-free survival (C) and prostate-specific antigen (PSA) progression-free survival (D). (Color version of figure is available online.) 
Table 2

Adverse events recorded during AA treatment (\%)

\begin{tabular}{|c|c|c|c|c|c|}
\hline$\%$ Grade $\geq 3$ & Belgian CU program & COU-AA-301 [1] & Sternberg et al. [15] & Caffo et al. [8] & Clayton et al. [9] \\
\hline Pain $^{\mathrm{a}}$ & 6.3 & 7.3 & - & - & - \\
\hline Diarrhea & 0 & 1.1 & - & 0.4 & 0 \\
\hline Nausea & 1.4 & 2.1 & - & 0.4 & 0 \\
\hline Vomiting & 0.5 & 2.7 & - & 0 & - \\
\hline Fatigue & 6.8 & 9.1 & - & 4.2 & 0.5 \\
\hline Peripheral edema & 0.8 & 0.5 & 1 & 0.4 & 0 \\
\hline Cardiac disorders $^{\mathrm{b}}$ & 0.8 & 5.2 & 2.2 & 0 & - \\
\hline Anemia & 13.9 & 7.8 & 1.7 & 4.2 & 0.1 \\
\hline Neutropenia & 1.9 & 0.1 & - & 0 & 1.1 \\
\hline Thrombocytopenia & 4.1 & 1.4 & - & 0.8 & 0 \\
\hline Liver enzymes & 3.5 & 3.8 & 8.1 & $0^{c}$ & 0.5 \\
\hline Hypokalemia $^{\mathrm{d}}$ & 7.3 & 4.4 & 1.2 & 0.4 & 1.6 \\
\hline Hypertension & 3.5 & 1.3 & 4.3 & 0.4 & 1.1 \\
\hline
\end{tabular}

${ }^{\text {a}}$ Pain was assessed by investigators in the Belgian compassionate use $(\mathrm{CU})$ program, whereas it was scored according to the Brief Pain Inventory in COU-AA-301.

${ }^{\mathrm{b}}$ Cardiac disorders included ischemic heart disease, myocardial infarction, supraventricular tachyarrhythmias, ventricular tachyarrhythmias, cardiac failure, and possible arrhythmia-related investigations, signs, and symptoms.

${ }^{\mathrm{c}}$ Any liver toxicity.

${ }^{\mathrm{d}}$ In the Belgian CU program at least 3 blood pressure measurements above threshold were needed to define hypertension.

better condition than patients outside clinical trials [6]. In our cohort, more patients had an ECOG status $\geq 2 \quad(25 \%)$ compared with COU-AA-301 (10\%). These patients were more susceptible to anemia and hypokalemia compared with patients with an ECOG status $<2$. Second, we used all parameters available in the patient files, unlike COU-AA-301 that used time-specific parameters. With more values available, we had a higher chance of detecting abnormalities. Third, because of ethical obligations in clinical trials, mild adverse events may be treated more aggressively than they would be in daily clinical practice, reducing the risk of exacerbation and thus the number of serious adverse events [7]. Fourth, patients in clinical trials generally demonstrate more rigorous compliance with both anti-tumor and other medication, reducing the chance of adherence-related side effects [17]. These data stress the ongoing need to screen patients for these specific side effects, especially in the first months of AA treatment that might allow timely intervention.

Fewer cardiac disorders were observed $(0.8 \%$ grade $\geq 3)$ compared with COU-AA-301 (5.2\%) [1]. Again, this might be explained by the different study design. Patients in COU-AA-301 underwent a standard cardiac examination, consisting of a multigated acquisition scan or cardiac ultrasonography and electrocardiography, at baseline and end-ofstudy and electrocardiography every 3 months during treatment [1]. Protocol-induced testing may lead to detection of cardiac disorders that would otherwise have gone undetected in daily clinical practice, where a cardiac examination is not routinely

Table 3

Oncological outcome with abiraterone acetate post-docetaxel as reported in literature

\begin{tabular}{|c|c|c|c|c|c|c|}
\hline & Background & $\begin{array}{l}N \\
\text { patients }\end{array}$ & $\begin{array}{l}\text { PSA response } \\
\text { rate }(\%)\end{array}$ & $\begin{array}{l}\text { Median time to PSA } \\
\text { progression, mo }\end{array}$ & $\begin{array}{l}\text { Median time to } \\
\text { radiographic } \\
\text { progression, mo }\end{array}$ & $\begin{array}{l}\text { Overall } \\
\text { survival (mo) }\end{array}$ \\
\hline Caffo et al. [8] & $\begin{array}{l}\text { Italian named patient program, } \\
\text { retrospective }\end{array}$ & 265 & 50 & NR & NR & 17 \\
\hline Clayton et al. [9] & $\begin{array}{l}5 \text { Canadian tertiary centers, } \\
\text { retrospective }\end{array}$ & 187 & 36 & 3.5 & NR & 11 \\
\hline Fizazi et al. [1] & $\begin{array}{l}\text { COU-AA-301 trial (active arm), } \\
\text { prospective }\end{array}$ & 797 & 29.5 & 8.5 & 5.6 & 15.8 \\
\hline Houédé et al. [10] & $\begin{array}{l}\text { French temporary authorization } \\
\text { for use program, retrospective }\end{array}$ & 306 & NR & NR & NR & 14.6 \\
\hline Sternberg et al. [15] & $\begin{array}{l}\text { Early access protocol trial in } \\
23 \text { countries, prospective }\end{array}$ & 2314 & NR & 8.5 & NR & NR \\
\hline $\begin{array}{l}\text { Van Praet et al. } \\
\text { (current) }\end{array}$ & $\begin{array}{l}\text { Belgian compassionate use } \\
\text { program, } \\
\text { retrospective }\end{array}$ & 368 & 37.4 or $29.4^{\mathrm{a}}$ & 4.1 or $6.0^{\mathrm{a}}$ & 5.8 & 15.1 \\
\hline
\end{tabular}

$\mathrm{NR}=$ not reported.

${ }^{\mathrm{a}}$ When using only 3-monthly PSA values. 
performed in the absence of symptoms [7]. Other AA-specific side effects, including elevated liver enzymes, peripheral edema and hypertension, were within expected ranges.

In our patient cohort, median OS was 15.1 months, which is in line with COU-AA-301 (15.8 mo) and other observational studies (11-17 mo) [1,8-10]. AA post-docetaxel appears to be similarly effective in daily clinical practice as in clinical trials: median time to radiographic progression $(5.8 \mathrm{mo})$ is comparable with COU-AA-301 (5.6 mo) [1]. Interestingly, PSA response rate was notably higher $(37.4 \%$ vs. $29.5 \%)$, whereas median time to PSA progression was notably shorter (4.1 vs. 8.5 mo) compared with COU-AA-301 [1]. However, unlike OS and radiographic progression, PSA kinetics are difficult to compare between both studies. In COU-AA-301 serum PSA was only measured at 3-monthly intervals. In our study, we used all available PSA values at least 4 weeks after AA start, which might more sensitively detect both PSA response and progression. When utilizing 3-monthly PSA measurements only, we found a PSA response rate of $29.4 \%$ and median time to PSA progression of 6.0 months, which is more in line with COU-AA-301. This phenomenon is consistent in the literature: other studies on real-world AA treatment restricting PSA testing to 3-monthly intervals had longer time to PSA progression (Sternberg et al.: 8.5 mo [15]) than studies using all PSA values clinically available (Clayton et al.: $3.5 \mathrm{mo}$ ) [9].

Limitations to the current analysis include its retrospective design with inherent missing data because of incomplete patient files and attribution bias. However, most outcomes were based on fixed data such as blood values or time points. As only patients treated within the Belgian $\mathrm{CU}$ program were included, these data might not be representative for every patient with MCRPC treated in daily clinical practice. Notwithstanding these limitations, this study gives important insights as these real-world data reflect what clinicians can encounter when starting a patient on AA post-docetaxel. Following COUAA-302, the place of AA in mCRPC treatment has shifted upfront to chemotherapy-naive patients. It seems unlikely that AA's tolerability would be inferior in the pre-docetaxel compared with the post-docetaxel setting, but evaluation of its realworld efficacy pre-docetaxel remains warranted.

\section{Conclusion}

These real-world data on AA efficacy are in line with COU-AA-301 and other reports in the post-docetaxel setting. Concerning tolerability, however, incidence of severe anemia and hypokalemia is up to $50 \%$ higher than reported in previous studies, indicating rigorous follow-up of these patients remains important.

\section{References}

[1] Fizazi K, Scher HI, Molina A, Logothetis CJ, Chi KN, Jones RJ, et al. Abiraterone acetate for treatment of metastatic castration-resistant prostate cancer: final overall survival analysis of the COU-AA-301 randomised, double-blind, placebo-controlled phase 3 study. Lancet Oncol 2012;13:983-92.

[2] Scher HI, Fizazi K, Saad F, Taplin ME, Sternberg CN, Miller K, et al. Increased survival with enzalutamide in prostate cancer after chemotherapy. N Engl J Med 2012;367:1187-97.

[3] de Bono JS, Oudard S, Ozguroglu M, Hansen S, Machiels JP, Kocak I, et al. Prednisone plus cabazitaxel or mitoxantrone for metastatic castration-resistant prostate cancer progressing after docetaxel treatment: a randomised open-label trial. Lancet 2010;376:1147-54.

[4] Parker C, Nilsson S, Heinrich D, Helle SI, O’Sullivan JM, Fossa SD, et al. Alpha emitter radium-223 and survival in metastatic prostate cancer. N Engl J Med 2013;369:213-23.

[5] Rathkopf DE, Smith MR, de Bono JS, Logothetis CJ, Shore ND, de Souza P, et al. Updated interim efficacy analysis and long-term safety of abiraterone acetate in metastatic castration-resistant prostate cancer patients without prior chemotherapy (COU-AA-302). Eur Urol 2014;66:815-25.

[6] Templeton AJ, Vera-Badillo FE, Wang L, Attalla M, De Gouveia P, Leibowitz-Amit R, et al. Translating clinical trials to clinical practice: outcomes of men with metastatic castration resistant prostate cancer treated with docetaxel and prednisone in and out of clinical trials. Ann Oncol 2013;24:2972-7.

[7] van Boxtel C, Santoso B, Edwards I. Drug benefits and risks: international textbook of clinical pharmacology—revised, 2nd ed. West Sussex, UK: John Wiley \& Sons Ltd, 2008.

[8] Caffo O, De Giorgi U, Fratino L, Lo ReG, Basso U, D’Angelo A, et al. Safety and clinical outcomes of patients treated with abiraterone acetate after docetaxel: results of the Italian Named Patient Programme. BJU Int 2015;115:764-71.

[9] Clayton R, Wu J, Heng DY, North SA, Emmenegger U, Hotte S, et al. A multicentre analysis of abiraterone acetate in Canadian patients with metastatic castration-resistant prostate cancer. Can Urol Assoc J 2014;8:E583-90.

[10] Houede N, Beuzeboc P, Gourgou S, Tosi D, Moise L, Gravis G, et al. Abiraterone acetate in patients with metastatic castrationresistant prostate cancer: long term outcome of the Temporary Authorization for Use programme in France. BMC Cancer 2015; 15:222.

[11] Available at: http://www.mskcc.org/nomograms/prostate/psa-doublingtime [accessed between 02.09.13. and 03.12.14.].

[12] Scher HI, Halabi S, Tannock I, Morris M, Sternberg CN, Carducci MA, et al. Design and end points of clinical trials for patients with progressive prostate cancer and castrate levels of testosterone: recommendations of the Prostate Cancer Clinical Trials Working Group. J Clin Oncol 2008;26:1148-59.

[13] Eisenhauer EA, Therasse P, Bogaerts J, Schwartz LH, Sargent D, Ford R, et al. New response evaluation criteria in solid tumours: revised RECIST guideline (version 1.1). Eur J Cancer 2009;45:228-47.

[14] Common Terminology Criteria for Adverse Events (CTCAE) version 4.03, published June 14, 2010. United States Department of Health and Human Services. Available at: http://evs.nci.nih.gov/ftp1/CTCAE/About. html.

[15] Sternberg CN, Castellano D, Daugaard G, Geczi L, Hotte SJ, Mainwaring PN, et al. Abiraterone acetate for patients with metastatic castration-resistant prostate cancer progressing after chemotherapy: final analysis of a multicentre, open-label, early-access protocol trial. Lancet Oncol 2014;15:1263-8.

[16] Azad AA, Eigl BJ, Leibowitz-Amit R, Lester R, Kollmannsberger C, Murray N, et al. Outcomes with abiraterone acetate in metastatic castration-resistant prostate cancer patients who have poor performance status. Eur Urol 2015;67:441-7.

[17] Gurwitz JH, Field TS, Harrold LR, Rothschild J, Debellis K, Seger AC, et al. Incidence and preventability of adverse drug events among older persons in the ambulatory setting. J Am Med Assoc 2003;289: $1107-16$. 